\section{0 \\ MANAGEMENT OF SEIZURES BY ACUTE HOSPITAL CLINICIANS AT THE END OF LIFE: A SERVICE EVALUATION}

Jennifer Inglis-Taylor, Mark Banting. University of Southampton, University Hospital Southampton

\subsection{6/spcare-2020-PCC.210}

Background Seizures are a distressing event, especially in patients at the end of life, when a significant proportion also lose the oral route for medications. Recently, an evaluation was done concerning medications Palliative Care physicians prescribe in these situations. This project aimed to evaluate how acute hospital clinicians manage seizures at the end of life. We also aimed to assess the knowledge of different routes of delivery for seizure medication at the end of life.

Methods Physicians within University Hospital Southampton (Stroke Medicine, Oncology and Neurology) were selected to take part in on online survey.

Results At the time of writing, there were 14 responses. Of the routes available for medication: $93 \%$ identified oral and IV, 71\% Subcutaneous and Per rectum, 36\% Nasogastric tube and 14\% PEG, Intramuscular, Buccal and Sublingual. The most frequently used route was oral. Of medications available subcutaneously, Midazolam was identified by $57 \%$, Levetiracetam by $29 \%$, Phenobarbitone by $21 \%$ and Clonazepam by $7 \%$. 71.4\% said that the consciousness of the patient affected their choice of medication. 29\% said they were 'not so confident' managing seizures at the end of life, but the rest were at least 'confident'. 86\% would contact Palliative Care for assistance.

Discussion/Conclusion Within this population, there is good knowledge of routes to use other than oral, but low incidence of use. Few doctors could identify many drugs available subcutaneously, and many were not confident in managing active seizures towards the end of life. Most would use the level of consciousness of the patient as a guide when prescribing. It is clear that educating physicians about options when the oral route is lost would be beneficial to confidence and patient care.

\section{1 'IRON'T YOU GOING TO GIVE A BLOOD TRANSFUSION?': ADDRESSING DEFICIENCIES IN ANAEMIA MANAGEMENT}

Sophie Jones, Nicola Loveday, Matthew Curtis. Rowans Hospice

\subsection{6/spcare-2020-PCC.211}

Background Anaemia is a common complication of advanced disease with associated symptoms having a negative impact on quality of life. A recent national hospice audit suggested a need for improvement in the management of anaemia in hospices, with a particular focus on investigation and more restrictive use of blood. As a result, we have updated local hospice guidelines and reviewed their use to assess the impact on patient care.

Method Alongside the introduction of updated guidelines, prospective data were collected from hospice inpatients with symptoms related to anaemia, who were being investigated for potential treatment. The data, collected over three months, included blood parameters to assess cause of anaemia and guide treatment options.
Results Of the seven patients investigated, four were folate deficient and five had either iron deficiency or functional iron deficiency.

Two were treated with folate replacement; one subsequently deteriorated rapidly. One was too unwell to be treated and the folate level improved in one without treatment. Four out of six patients (one not measured) had reduced serum iron yet ALL ferritin results were $>30 \mathrm{microgram} / \mathrm{L}$ and five had a ferritin $>1000$ microgram/L (range 186-3383). CRP results ranged from 95 to 206 .

Conclusion This case series highlights the complexity of interpreting results to guide management of anaemia in the hospice inpatient population. When a deficiency is identified, treatment with folic acid or iron carries less risk than treatment with blood. However, with ferritin levels this high, treatment with oral or intravenous iron is not usually recommended. Iron may not represent a frequently useful treatment option in this population. We are not aware of evidence confirming a positive clinical response to these treatments in hospice patients with complex underlying aetiologies contributing to anaemia. We believe this highlights a need for research to demonstrate clinical benefit to support these treatments.

\section{2 ANTICOAGULATION IN PALLIATIVE CARE: A MULTI-SITE REGIONAL AUDIT OF CLINICAL PRACTICE}

Sara McLintock, Kat Moss, Grace Ting, Simon Roughneen, Amanda Watson, Claire Cadwallader, Jessica Dodd, Alison Coackley. Marie Curie Hospice Liverpool, Liverpool University Hospitals NHS Foundation Trust, The Clatterbridge Cancer Centre NHS Foundation Trust, Warrington and Halton Teaching Hospitals NHS Foundation Trust

\subsection{6/spcare-2020-PCC.212}

Background The Palliative Care population represents a heterogenous group of patients with variable risk factors for bleeding and venous thromboembolism (VTE). Decisions about VTE treatment and primary prophylaxis can be challenging in these patients.

Aim The aim of this audit was to evaluate the management of anticoagulation in the hospice, hospital and community settings against current guidelines. The results of this audit, in addition to an extensive systematic review, informed the update of regional guidelines for the management of anticoagulation in Palliative Care Patients.

Methods A retrospective case note review was carried out across a regional palliative care network in North West England.

Results 189 patient records were analysed across 12 different sites within the region. $70 \%$ of patients were in hospice, $23 \%$ in hospital and $7 \%$ in the community. Of 89 patients on treatment dose anticoagulation, only $9 \%$ had the intended duration of anticoagulation documented. Of 60 patients on treatment dose low molecular weight heparin (LMWH), weight was documented in 63\%. Renal function was documented in $88 \%$ of patients on LMWH, and in $75 \%$ of 24 patients on treatment dose direct oral anticoagulants (DOACs). $71 \%(66 / 93)$ had a venous thromboembolism assessment completed. Prophylactic anticoagulation was given in $43 \%$ of these 93 patients. For prophylaxis, the majority (90\%) received LMWH, with the remaining $10 \%$ prescribed a DOAC. 42 patients died during the episode of care. The anticoagulation (either primary prophylaxis or treatment) was continued until death in $43 \%$ of these patients.

Conclusion Clear documentation of anticoagulation duration, and factors which influence choice of anticoagulant and dose 